\title{
Dubowitz Score
}

National Cancer Institute

\section{Source}

National Cancer Institute. Dubowitz Score. NCI Thesaurus. Code C81282.

The numerical value that represents a clinical assessment of neurological and physical criteria, performed between birth and five days of age, to determine an infant's gestational age and maturity. 